\title{
Positioning ACM for an Open Access Future
}

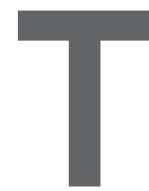

HE AGE OF open access is upon us. Increasingly, the consensus of authors of research articles and their funding institutions is that the fruits of taxpayer-supported research should be freely available to the public. This is a compelling argument and a noble goal.

But, achieving open access is not easy. Professional maintenance and distribution of large digital archives, guaranteed for the long term, does incur significant cost. The most promising model for recovering such costs under an open-access regime is an authorpays (or, in effect, a funding institution pays) model. Such a scheme introduces issues of its own. If publishers generate revenue by producing more content (paid for by authors) rather than quality content (paid for by subscribers), then the natural tendency in the system will be for the generation of large quantities of low-quality content. Indeed, we have seen the rise of predatory publishers, actively seeking authors to pay for publication in venues devoid of the exacting scrutiny of conscientious peer review. The result is a glut of third-rate publications that add noise rather than insight to the scientific enterprise.

The important question is: Can we establish a sustainable economic model for publication that serves the interest of both authors and the reading public? We submit that non-profit professional societies must play a critical role in this regard. They are the hallmark of quality in publications, and must remain so to serve the interests of the reading public. But, how do we transition from the current subscription model to a new financial model enabling open access in a way that does not bankrupt the organization in the process? This question has occupied the attention of the ACM Publications Board for several years. Because the stakes are high, the Board has chosen to move with caution.

Because we do not have a reliable crystal ball, we have chosen to provide an array of options for ACM authors and Special Interest Groups (SIGs) to enable a natural, slow, and (hopefully) stable evolution of the publication en- terprise into the future. Examples of this are ACM's long-standing policies enabling author-produced versions of ACM-published materials to be posted on author Web pages, on the pages of their institutions, and on archives mandated by funding institutions. A shining example of such green openaccess policies is ACM's Author-Izer service, which allows authors to place specialized links on their Web pages that tunnel through ACM's paywall to provide free access to the definitive versions of their papers, while capturing download statistics displayed in the ACM Digital Library (DL).

In the next few months ACM will roll out more options for authors and SIGs, which will provide even greater levels of flexibility with regard to open access.

1. Author-pays Open Access Option. Individual authors (or their institutions) will have the option to pay a fee that covers ACM publication and curation costs at the time of publication, after which the article will be freely available via the ACM DL platform. The fee, which has yet to be set, will differentiate between journal articles and conference papers, as well as between ACM members and non-members.

2. Open Access During the Period Around SIG Conferences Option. SIGs will have the option to make the proceedings from their conferences freely available via the ACM DL platform for up to two weeks before the event and up to two weeks after. Not only will this option facilitate easy access to the proceedings by conference attendees, it will also enable the community at large to experience the excitement of learning about the latest developments being presented in the period surrounding the event itself.

3. Open Access for Most Recent Instance of SIG Conferences Option. SIGs will have the option to maintain tables-of-contents for the most recent instance of its conferences on the conference website with ACM AuthorIzer links that provide free access to the definitive version of the article maintained in the ACM DL. For conferences that are not in a recurring series, such access will be enabled for up to one year. When selected by the sponsoring SIG, this reverse embargo will provide open access to conference papers during the period in which they are of greatest interest.

4. Options for Rights Management. When publishing articles with ACM, authors will have three options for the management of publication rights for their work. Authors who desire the convenience of having ACM maintain rights and permissions associated with their works will continue to be able to execute copyright transfer to ACM. ACM's copyright policy will continue to grant authors liberal rights for the reuse of their work and the posting of personal versions. Authors who prefer to retain copyright or want a more explicit publishing contract will have the option of signing one of two license agreements. The first will have terms similar to that of the existing copyright transfer. However, this option will provide increased clarity about the rights of both parties. Finally, those authors who wish to retain all rights to their work can do so by exercising the author-pays open access option described previously. In that case all rights remain with the author and ACM is simply granted a permanent license to distribute.

The set of changes unveiled here are but another step in an ongoing process in which ACM adapts to the new realities of scholarly publishing. Many further refinements are possible. For example, if the author-pays option is successful, the Publications Board may consider the feasibility of making previously published papers in the DL open access, or making entire proceedings open access using funding from registration fees or sponsors. As the Board considers each change it will continue to be responsive to the needs and wishes of the ACM community while fulfilling its responsibility to maintain a healthy and sustainable publications program. C

Ronald F. Boisvert and Jack W. Davidson are co-chairs of the ACM Publications Board.

(C) 2013 ACM 0001-0782/13/02 